\title{
Analog simulation on the Frequency Character of Double Chambers Self-excited Oscillation Pulsed Water Jet Nozzle
}

\author{
Jianghong $\mathrm{Pei}^{1}$ Zhangwei $\mathrm{Bai}^{2}$ \\ 1Chongqing Industry Polytechnic College, Chongqing 401120, China \\ pjh_cq@163.com \\ 2 Chongqing Vocational Institute of Engineering, Chongqing 402260, China \\ 89441486@qq.com
}

\begin{abstract}
Keywords: Double chamber, Self-excited oscillation, Pulsed water jet nozzle, Natural frequency
Abstract. Based on the theory of fluid network, the mathematical model of the self-excited oscillation pulsed water jet device was established, theory analyses the frequency characteristic of self-excited pulsed water jet nozzle on double chambers. The result shows that structural parameters greatly affect natural frequency of the device. And also, there has the optimized range among structural parameters which is different from the range of singlet chamber nozzle. lastly, self-excited pulsed water jet nozzle of double chambers can improved the surge pressure higher than singlet chamber nozzle in the same condition. The result is useful to the design of pulsed jet nozzle.
\end{abstract}

\section{Introduction}

Self-excited oscillation pulsed jet is a new type of high efficient pulse jet, compared with other pulse jet it has simple structure, without excitation source, sealed without moving parts, etc[1-3]. It has been widely used in cleaning, broken rocks, buildings, cutting and auxiliary mining and other industries. The mechanism of self-excited oscillation is that, firstly, when the jet or shear flow flows to the downstream process in the shear layer, due to the instability of the shear layer has the effect of amplification to some vorticity, which leads to a certain frequency vorticity is amplified, and formed a series of discrete vortex ring. Secondly, when the vortex ring collided with the wall collision, it leads to pressure disturbance wave formatted and which spread to the upstream in the form of sound velocity, it induced new vorticity disturbance produced. When the pressure disturbance wave spread to upstream, it results in wave fluctuating along crosswise greatly in collision zone around the shear layer, when its spread to jet core layer makes the fluid impedance of the chamber exit appeared periodic change. Lastly, the self-excited oscillation pulsed jet formatted[4-5]. Experiments show that the peak pressure of self-excited oscillation pulsed jet can be increased by $15 \% \sim 20 \%[6]$. How to further improve the pulse jet peak pressure, it has become research hot spot. But it is focused on getting the best resonance effect by the optimization of single chamber self-excited oscillation cavity structure and of upper and lower nozzle aperture size, to improve the peak pressure pulse jet[7]. This paper makes a beneficial exploration on the double chamber of self-excited oscillation nozzle, in order to theoretically explore the self-excited oscillation of dual chamber nozzle can get higher peak pressure than single chamber self-excited oscillation injection nozzle or not, so that services for the engineering practice. its structural model diagram is shown in figure 1. According to similarity theory, analyzed the relation among the structure parameters on the double chamber self-excited oscillation pulsed device, pressure peak and the inherent frequency of nozzle.

\section{Double chamber self-excited oscillation nozzle model building}

According to the fluid neural network theory[8], mass flow rate compared with electric current, pressure compared with voltage, flow resistance compared with resistance[8]. And, the lumped parameter model on double chamber of self-excited oscillation pulsed jet nozzle was built by similarity theory (as shown in figure 2). In this model, $\mathrm{R}_{1}$ and $\mathrm{R}_{2}$ is the fluid resistance of the up nozzle and the down nozzle respectively. $\mathrm{L}$ is the fluid inductance of the up pipeline. $\mathrm{C}_{1}$ and $\mathrm{C}_{2}$ is the fluid capacitance 
of the first oscillate chamber and the second oscillate chamber, respectively. According to the quasi-stationary assumption theory of fluid network, the small disturbance signal of the turbulent flow resistance is nonlinear, so the dynamic resistance is shown as the following:

$$
R=\frac{\delta(\Delta p)}{\delta Q}=\frac{v \sqrt{\zeta}}{A C_{f}}
$$

Where: $v$ is the fluid average velocity, $\zeta$ is the coefficient of local resistance of nozzle, $A$ is the sectional area of nozzle, $C_{f}$ is the discharge coefficient of flow nozzle.

$$
C=\left.\frac{\partial M}{\partial p}\right|_{V=\mathrm{C}}=\frac{\pi D^{2} L}{4 a^{2}}
$$

Where: $D$ is the diameter of oscillate chamber, $L$ is the length of oscillate chamber, $a$ is the fluid wave velocity in the oscillate chamber.

$$
L=\frac{\Delta p}{d Q / d t}=\frac{4 l_{0}}{\pi d_{0}^{2}}
$$

Where: $l_{0}$ is the pipe length of up nozzle, $d_{0}$ is the pipe diameter of up nozzle.

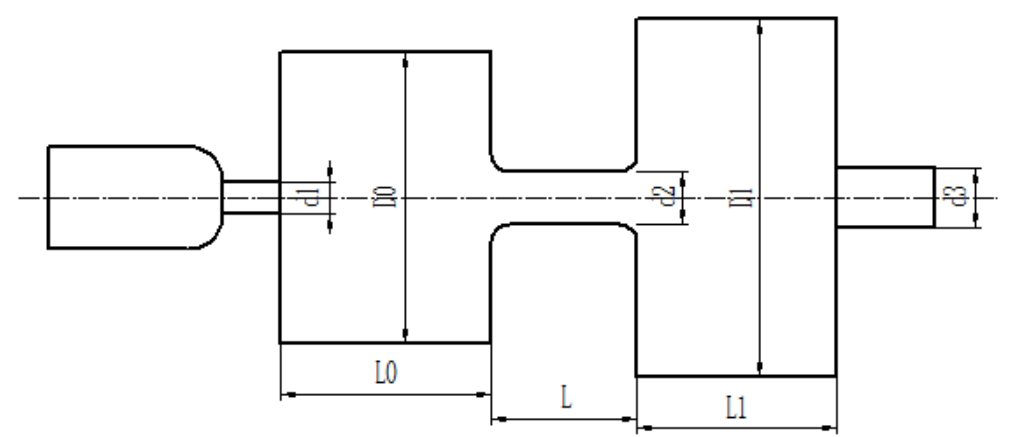

Fig. 1 The model of double chambers self-excited oscillation pulsed water jet nozzle

In this model, where $d_{1}$ is diameter of up nozzle; $D 0$ is the diameter of the first oscillation chamber; $L 0$ is the length of the first oscillation chamber; $d_{2}$ is the diameter of connecting pipe between oscillation chamber; $D 1$ is the diameter of the second oscillation chamber; $L 1$ is the length of the second oscillation chamber; $d_{3}$ is the diameter of down nozzle.

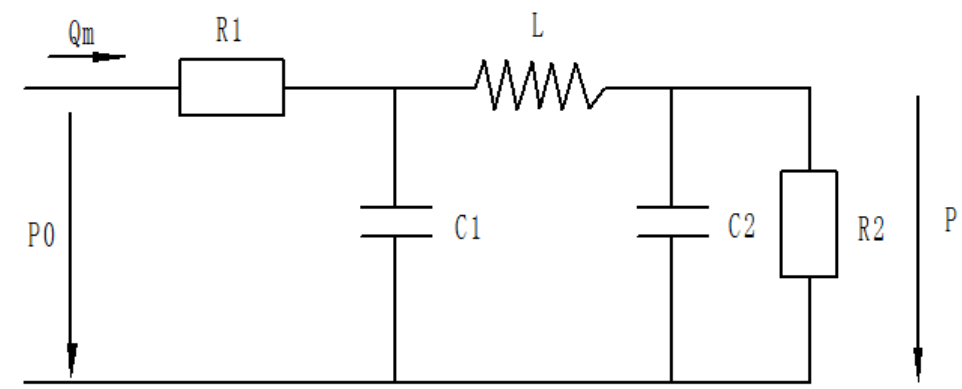

Fig.2 lumped parameter net model of double chambers self-excited oscillation pulsed water jet nozzle

In this model, where $P 0$ is the inlet pressure of up nozzle; $P$ is the outlet pressure of down nozzle. The impedance is made up of components $L, C 2$ and $R 2$ :

$$
Z 1=j w L+\frac{R 2 \times \frac{1}{j w C 2}}{R 2+\frac{1}{j w C 2}}
$$

The total impedance of the overall system 


$$
Z=R 1+\frac{Z 1 \times \frac{1}{j w C 1}}{Z 1+\frac{1}{j w C 1}}
$$

The pressure drop through $R 2$

$$
p=\frac{\frac{p_{0}}{Z} \cdot \frac{\frac{Z 1}{j w C 1}}{\frac{1}{j w C 1}+Z 1} \cdot \frac{\frac{R 2}{j w C 2}}{\frac{1}{j w C 2}+R 2}}{j w L+\frac{\frac{R 2}{j w C 2}}{\frac{1}{j w C 2}+R 2}}
$$

By the above formula

$$
\frac{p}{p_{0}}=\frac{R 2}{R 1+R 2} \cdot \frac{1}{1-w^{2} L C 1+j w(D-E)}
$$

Where

$$
\begin{aligned}
& D=\frac{2 L+C 1 \times R 1 \times R 2}{2(R 1+R 2)} \\
& E=\frac{w^{2} \times C 1 \times C 2 \times R 1 \times R 2}{R 1+R 2}
\end{aligned}
$$

frequency response function、 amplitude-frequency and phase frequency characteristics of the system is

$$
\begin{aligned}
& G(s)=\frac{R 2}{R 1+R 2} \cdot \frac{1}{1-w^{2} L C 1+s[D-E]} \\
& A(w)=\frac{1}{\sqrt{\left(1-w^{2} L C 1\right)^{2}+w^{2}[D-E]^{2}}} \\
& \varphi(w)=-\operatorname{arctg} \frac{w[D-E]}{1-w^{2} L C 1}
\end{aligned}
$$

Imaginary part of the equation (7) is zero, which is condition of self-exciting oscillation coming into being

$$
\frac{2 L+C 1 \times R 1 \times R 2}{2(R 1+R 2)}=\frac{w^{2} \times C 1 \times C 2 \times R 1 \times R 2}{R 1+R 2}
$$

Natural frequency can be obtained by equation (8)

$$
w=\sqrt{\frac{2 L+C 1 \times R 1 \times R 2}{2 C 1 \times C 2 \times R 1 \times R 2}}
$$



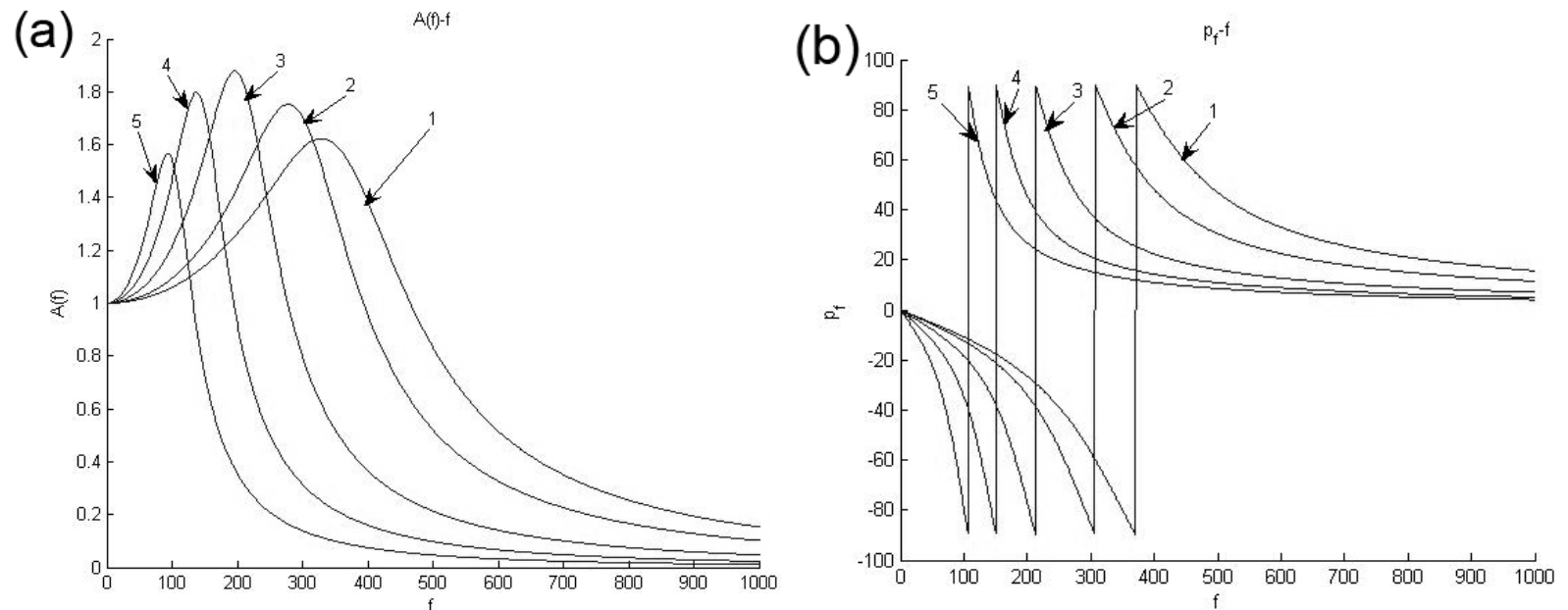

(a)The amplitude frequency characteristics on different chamber length

(b)The phase-frequency characteristic under different chamber length

Fig.3 The frequency characters on different chamber length

$\begin{array}{ll}\text { 1. } L_{0} / d_{1}=L_{1} / d_{2}=2.4 & \text { 2. } L_{0} / d_{1}=L_{1} / d_{2}=3.5 \text { 3. } L_{0} / d_{1}=L_{1} / d_{2}=7.25\end{array}$

4. $L_{0} / d_{1}=L_{1} / d_{2}=14.5$ 5. $L_{0} / d_{1}=L_{1} / d_{2}=29$

\section{Result and analysis}

In the design process of the double chamber self-excited oscillation pulsed nozzle, the experience of the single chamber self-excited oscillation pulsed nozzle is referenced[1,5]. The surface of the upper nozzle is streamline, and the surface of the down nozzle is circular hole. $P_{0}=10 M P a 、 d_{1}=2 \mathrm{~mm}$ 、 $d_{2} / d_{1}=1.2 、 d_{3} / d_{2}=1.2 、 D_{0} / d_{1}=20 、 D_{1} / d_{2}=20$ and $l / d_{2}=10$, which is substituted into equation (1), (2), (3), (9) and (10). And we can get the amplitude-frequency and phase-frequency characteristics characters on different chamber length and chamber diameter(see figure 3 and figure 4). When calculating the amplitude frequency characteristics, the wave velocity $a$ is $500 \mathrm{~m} / \mathrm{s}$. that is the cavitation phenomenon would produced in the self-excited oscillation chamber, and the steam pocket will be formed in the middle of the jet area, which will reduce the fluidic wave velocity. Fig. 3 shows that double chamber self-excited oscillation pulsed jet nozzle has the low-pass filtering booster function and pressure boost characteristics. There is a low passband in the nozzle system, when the jet frequency is in the low passband, and jet can produce pressure boost. So, designing a jet nozzle, we should make the nature frequency of nozzle near main frequency of jet, which is very important.
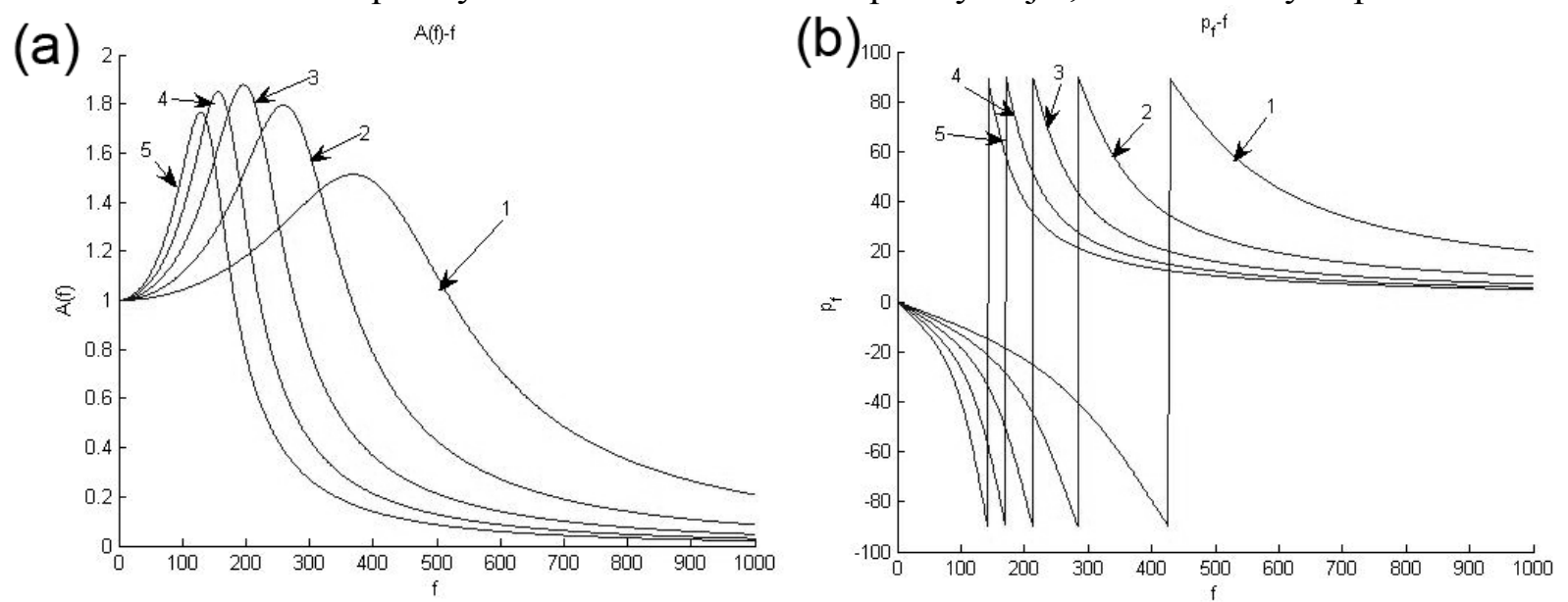

(a)The amplitude frequency characteristics on different chamber diameter

(b)The phase-frequency characteristic under different chamber diameter

Fig.4 The frequency characters on different chamber diameter 

$D_{0} / d_{1}=D_{1} / d_{2}=30$
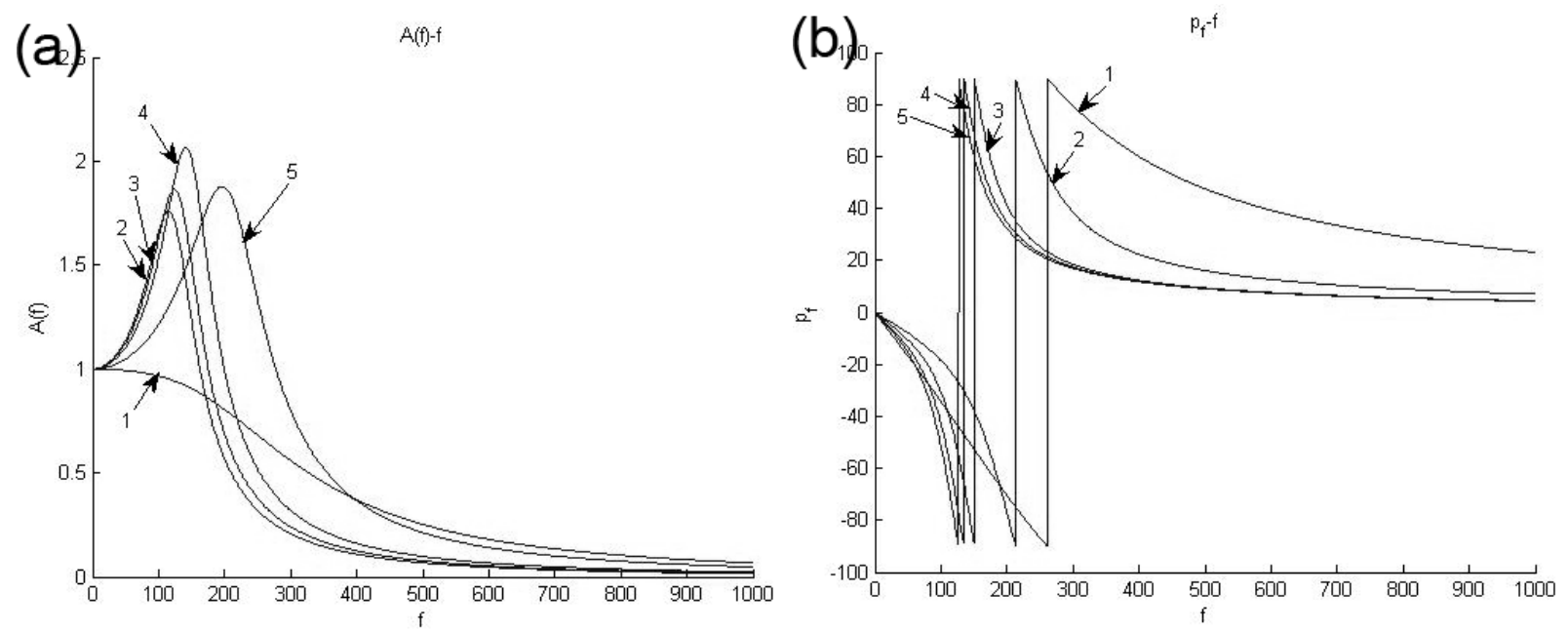

(a)The amplitude frequency characteristics on different nozzle rate

(b)The phase-frequency characteristic on different nozzle rate

Fig. 5 the frequency character on the different diameter ratio of nozzle

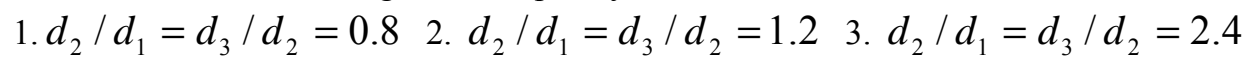

4. $d_{2} / d_{1}=d_{3} / d_{2}=3$ 5. $d_{2} / d_{1}=d_{3} / d_{2}=3.4$

The Fig. 3 shows: the amplitude of the system increase and then decrease with the length of the self-excited oscillation chamber, there is an optimal length of the oscillation chamber on double chamber corresponding to the amplitude of the system $\left(L_{0} / d_{1}=7.25, L_{1} / d_{2}=7.25\right)$, the optimal length of the oscillation chamber is different from single oscillation chamber nozzle[1,3,6]. The jetting peak pressure increase of $87.8 \%$, which shows double chamber oscillation jetting nozzle has a better pressure increase than single chamber oscillation jetting nozzle. The resonance frequency of the double chamber jetting nozzle decreases with the length of the self-excited oscillation chamber. The nature frequency corresponding to the resonance peak on double chamber jetting nozzle is lesser than that of single chamber jetting nozzle.According to the double chamber nozzle parameters network model, the total impedance of double chamber oscillation jetting nozzle is lesser than single chamber oscillation jetting nozzle, which leads resonance frequency of double chamber oscillation jetting nozzle is lesser than single chamber oscillation jetting nozzle. The phase-frequency of double chamber jetting nozzle has the same change rule. Phase lag Angle will be increased as the frequency increases. when the frequency exceeds the resonant frequency, phase advance angle will be decreased as the frequency increases. This is because, when the frequency is small, system impedance is flow capacitive impedance, so there is phase lag; when the frequency is over resonant frequency, system impedance is flow perceptual impedance, so there is phase advance. The frequency corresponding to step change is the natural frequency.

The Fig. 4 shows the system natural frequency decreases as oscillation chamber diameter increase, system low transmission bands narrows down as oscillation chamber diameter increase. The optimal chamber diameter rate is $D_{0} / d_{1}=20, D_{1} / d_{2}=20$.

The Fig. 5 shows system natural frequency decreases as the Upper and lower nozzle ratio increases; system transmission bands increases and then decreases as the Upper and lower nozzle ratio increases. The optimal Upper and lower nozzle ratio is $d_{2} / d_{1}=d_{3} / d_{2}=3$, which is much higher than the optimal diameter rate of single nozzle. 


\section{conclusion and prospect}

1) By theoretical model analyzing, double chamber self-oscillation jetting nozzle can improve peak pressure greatly than single chamber self-oscillation jetting nozzle.

2)The device has a low pass filter and pressure resonance effect, there is the maximum resonant peak pressure near the natural frequency.

3 ) The cavity length, cavity diameter and Upper and lower nozzle ratio of nozzle has a great influence on the natural frequency. The maximum resonant peak pressure corresponding the optimal system structure parameter, there are great different from the optimal system structure parameter between double chamber and single chamber nozzle.

4 ) The frictional resistance loss and local resistance loss on double chamber self-excited oscillation nozzle is great than single chamber self-excited oscillation nozzle.

\section{Acknowledgements}

This work was financially supported by the Natural Science Foundation Project of CQ CSTC item(cstc2012jjA00004)

\section{References:}

[1] LI Xiao hong, YANG Lin, WANG Jian sheng, etc. The natural frequencies of the self-excited oscillation pulsed jet device features[J]. Journal of china coal society, 2000 , 25(6) : 641-644

[2] WANG Le-Qin, WANG Xun-Ming, XU Ru-Liang. The experimental study on the structural parameters ration of self-excited oscillation pulsed nozzle[J]. Journal of Engineering Thermophysics, 2004 , 25(6) : 956-958

[3] TANG Chuan-lin,HU Dong,PEI Jiang-hong. The experimental research on the dynamic characteristics of self-excited oscillation pulsed jet[J]. Water Resources and Hydropower Engineering, 2006 , 37(12) : 71-74

[4] Pei Jianghong, Tang Chuanlin, Zhang fenghua, etc. Theory and Experiment of the Self-excited Oscillation Pulsed Jet Nozzle in the Non-submerged Jet Condition[J]. Mining \& Processing Equipment, 2006 , 35(10):96-98

[5] YANG Lin, LI Xiao hong, WANG Jian sheng. The influence of inherent frequency with structural parameters on self-excited oscillation pulsed jet[J]. Fluid Machinery, 2001 , 29(2):26-28

[6] TANG Chuan-lin,HU Dong,PEI Jiang-hong. The experimental study on the frequency of self-excited oscillation nozzle[J]. Acta Petrolei Sinica, 2007 , 28(4) : 122-125

[7]LI Jiang-Yun, WANG Le-Qin, XU Ru-Liang, etc. The cavitation model on self-excited pulsed jet of Low voltage large diameter nozzle[J]. Journal of Engineering Thermophysics, 2005 , 26(3) : 438-440

[8] LUO Zhichang. Fluid network theory[M]. Mechanical Industry Press, 1988 